\title{
An Automated Velocity Dealiasing Scheme for Radar Data Observed from Typhoons and Hurricanes
}

\author{
GUANGXIN HE \\ Key Laboratory of Meteorological Disaster, Ministry of Education (KLME), and International Joint Research \\ Laboratory on Climate and Environment Change (ILCEC), and Collaborative Innovation Center on Forecast and \\ Evaluation of Meteorological Disasters, and Center of Data Assimilation for Research and Application, Nanjing \\ University of Information Science and Technology, and Nanjing Joint Center of Atmospheric Research, Nanjing, \\ and Key Laboratory of Meteorology and Ecological Environment of Hebei Province, Shijiazhuang, China, \\ and National Center for Atmospheric Research, Boulder, Colorado \\ JUANZHEN SUN AND ZHUMING YING \\ National Center for Atmospheric Research, Boulder, Colorado
}

(Manuscript received 21 September 2017, in final form 15 November 2018)

\begin{abstract}
Accurate and automated dealiasing of radar data is important for data interpretation and downstream applications such as numerical weather prediction (NWP) models. In this paper an improved radial velocity dealiasing scheme is presented and evaluated using observations from several S-band radars under the severe weather conditions of typhoons and hurricanes. This scheme, named Automated Dealiasing for Typhoon and Hurricane (ADTH), is a further development of the China New Generation Doppler Weather Radar (CINRAD) improved dealiasing algorithm (CIDA). The upgraded algorithm ADTH includes three modules designed to select the first radial from which the dealiasing process starts, to conduct a two-way multipass dealiasing, and to perform an error check for a final local dealiasing. The dealiasing algorithm is applied to two typhoon hurricane cases and four typhoon cases observed with radars from CINRAD, NEXRAD of the United States, and the Taiwan radar network for a continuous period of $12 \mathrm{~h}$ for each of the selected cases. The results show that ADTH outperforms CIDA for all of the test cases.
\end{abstract}

\section{Introduction}

Doppler radar observations have high spatial and temporal resolutions and have been used for many meteorological applications, ranging from providing operational radar products for the prediction of adverse and hazardous weather systems to initializing numerical weather prediction (NWP) models. Radial velocity observed from Doppler weather radar plays an important role in radar data assimilation and has considerable potential to improve precipitation predictions (e.g., Sun 2005; Sun and Zhang 2008; Gao et al. 2004; Hu et al. 2006; Xiao and Sun 2007; Simonin et al. 2014). The assimilation of these data into NWP models requires accurate and fully automated quality control of the radar data. One of the longstanding challenges for the quality control of Doppler

Corresponding author: Dr. G. He, ghe@nuist.edu.cn radar radial velocity measurements is the correction of aliased velocities.

The maximum observed velocity, or Nyquist velocity $V_{\text {max }}$, from the Doppler radar data is related to the pulse repetition frequency (PRF) of the radar with a transmitted wavelength $\lambda$. It is defined as

$$
V_{\max }=\frac{(\mathrm{PRF}) \lambda}{4} .
$$

The interval $\left[-V_{\max }, V_{\max }\right]$ refers to the unambiguous velocity interval. As shown in Eq. (1), $V_{\max }$ can be increased by increasing the PRF. Nevertheless, since the maximum unambiguous range $R_{\max }$ is inversely proportional to the PRF, a larger PRF will reduce the $R_{\max }$. The trade-off between the $V_{\max }$ and $R_{\max }$ leads to the limited values of the Nyquist velocity. Any real radial velocity not in the interval of $\left[-V_{\max }, V_{\max }\right]$ will appear within $\pm V_{\max }$ and thus will be aliased. The relationship 
between the true radial velocity $V_{T}$ and the observed velocity $V_{O}$ is as follows:

$$
V_{T}=V_{O}+2 n \times V_{\max },
$$

where $n$ is an integer. An effective radial velocity dealiasing algorithm can recover $V_{T}$ from the raw radar observation $V_{O}$ by choosing the proper $n$.

Since the 1970s, many dealiasing algorithms have been developed. These algorithms are mainly based on two general techniques, namely, the continuity check method and the reference wind check method. Although some success has been demonstrated, accurate velocity dealiasing remains a challenge. In particular, when the radial velocity is assimilated into an operational NWP model, it is necessary to have a dealiasing scheme that is fully automated and highly reliable. A small percentage of failed or erroneously dealiased data can cause analysis errors in the model's initial conditions and hence contaminate the subsequent forecasts.

The reference wind check method uses a reference wind field to dealias the radial velocity at each grid point individually (Gao et al. 2004; Lim and Sun 2010; Xu et al. 2011). As such, it has the advantage of being unaffected by any erroneously dealiased data of its neighboring points, avoiding the spatial spreading of such an error, which can occur in algorithms based on the continuity check method. However, its disadvantage is that the method can fail for grid points where the reference winds have large errors. The first continuity check algorithm, introduced by Ray and Ziegler (1977), was onedimensional, in which only radial gate information was used to correct the aliased velocities along each radial. Following their work, several two-dimensional algorithms (Merritt 1984; Bergen and Albers 1988; Eilts and Smith 1990; Jing and Wiener 1993; Gong et al. 2003; Gao et al. 2004; Zhang and Wang 2006; He et al. 2012b) that dealiased in both the azimuthal and radial directions were developed. Some algorithms were combined with a reference wind check method in which additional information from a radiosonde or velocity-azimuth display (VAD) wind profile (Tabary et al. 2001) was used for the dealiasing of isolated areas (Merritt 1984; Eilts and Smith 1990; Gong et al. 2003; Gao et al. 2004; $\mathrm{Xu}$ et al. 2011). Based on a two-dimensional dealiasing algorithm, James and Houze (2001) developed a fourdimensional algorithm by adding information from different elevation angles and volume scans. Most of the algorithms are designed for a Nyquist velocity between 20 and $36 \mathrm{~m} \mathrm{~s}^{-1}$ but tend to fail for some typhoon and hurricane cases when multiple-aliased velocity observations appear. A series of algorithms (Wang et al. 2012; Xu et al. 2014; Jiang and Xu 2016) were developed for aliased radar radial velocities obtained from a hurricane and a typhoon. The algorithms by Xu et al. (2014) and Jiang and $\mathrm{Xu}$ (2016) are built on alias-robust velocityazimuth display analysis (Xu et al. 2010).

He et al. (2012a, hereafter HE2012a) proposed an upgraded two-dimensional continuity check scheme for dealiasing the S-band China New Generation Doppler Weather Radar (CINRAD) observations, named the CINRAD improved dealiasing algorithm (CIDA). A novel feature of CIDA was the use of a new method to select the initial reference radial along a "zero line" where the radial velocities are very small and less likely to be aliased. In addition, a multipass procedure in CIDA enabled the improved dealiasing of aliased velocity echoes adjacent to missing data, which also eliminated the need for VAD wind profiles or radiosonde. The applications of the algorithm to multiple cases of typhoons, squall lines, and heavy rains observed by CINRAD showed superior performance to those of two standard NEXRAD (U.S. radar network) algorithms (HE2012a).

Although HE2012a has made significant contributions toward our goal to develop a fully automated, accurate radar velocity dealiasing scheme that is applicable for different weather system observation, the question remains whether the scheme is generally applicable for radar measurements from all the typhoon and hurricane cases. Also, HE2012a tested CIDA on only one selected volume for each of the selected weather cases. Our experience with CIDA showed that the algorithm could fail in some typhoon and hurricane situations when, for example, multiple aliases occurred, the typhoon or hurricane eye was very close to radar station, or aliased velocities were surrounded by missing data. Therefore, improvements are required for the algorithm to be capable of and robust enough for dayto-day real-time observations. In the current study, we improved the dealiasing algorithm of HE2012a and it is referred to as Automated Dealiasing for Typhoon and Hurricane (ADTH). We implemented improvements in three areas with the goal of developing a fully automated, accurate radar velocity dealiasing scheme for real-time typhoon and hurricane observations. ADTH was applied to radar data from several different typhoon and hurricane cases to examine its robustness. We ran ADTH continuously for $12 \mathrm{~h}$ for each of the selected cases to evaluate the scheme's robustness with large samples of observations and to obtain statistical verification. In these cases, more than $75 \%$ of the raw observed data have aliased velocities. To quantify the improvement of ADTH over CIDA, all of the same data were run through CIDA.

The ADTH described in this paper includes three new developments. First, the refinement of the first radial selection method using winds estimated from the radial 
velocity as an additional constraint; second, the upgraded multipass dealiasing algorithm added passes along the azimuthal direction; third, an improved least squares error check method to perform localized dealiasing.

The rest of the paper is organized as follows. Section 2 describes the details of the modules in the ADTH algorithm. Section 3 provides the dealiasing results of four typhoon cases and two hurricane cases and the results of their statistical analyses. Finally, section 4 concludes with a brief summary.

\section{Description of the ADTH algorithm}

The improved dealiasing algorithm ADTH consists of three modules: 1) Selection of the first radial. This criterion is based on the gradient velocity-azimuth display (GVAD) wind to reduce the failure rate in typhoon/hurricane cases, especially when multiple-aliased velocities appear. 2) Dealiasing in radial and azimuthal directions. This includes a two-way dealiasing procedure in both radial and azimuthal directions to improve the dealiasing reliability, especially when the typhoon/hurricane eye is very close to a radar station. 3) The third is local error checks. This includes a local error check and a linear square error check in the radial direction and a quadratic least squares error check in the azimuthal direction. These three modules are described in detail below. Attention will be paid to our improvements over the original scheme in HE2012a in the following description. Among the three modules, modules 1 and 3 are most sophisticated with new methodologies; thus, three figures (Figs. 1 and 4, and 5) are provided for these modules to facilitate the description.

\section{a. Module 1: Selection of the first radial}

\section{A CRITERION BASED ON THE GVAD WIND TO REDUCE THE FAILURE RATE IN TYPHOON/ HURRICANE CASES, ESPECIALLY WHEN MULTIPLE-ALIASED VELOCITIES APPEAR}

With the limitation of hardware performance of the radar station, there will be some range folding gates appearing in low-elevation angles of the radial velocity field. All of the range folding from different radar observations will be abandoned and set up with missing data before dealiasing. This step will not change the value of valid gates and will not affect the performance of our dealiasing algorithm.

Correctly selecting the first radial such that the radial velocities do not contain aliased data is critical for dealiasing algorithms to work successfully, as the subsequent dealiasing depends on the previously corrected radial velocity. Because the radial velocities along the radial where the atmospheric wind is nearly perpendicular to the radar beam are very small (or near zero) and are therefore less likely to be aliased, CIDA finds the zero line (illustrated by the yellow line in Figs. 1a and 1b) by computing the mean radial velocity averaged along each radial (blue curves in Figs. 1c and 1d). Also plotted in Figs. $1 \mathrm{c}$ and $1 \mathrm{~d}$ are the total numbers of the valid gates used in the calculations of the means (red curves). The first radial should have a local minimum average of mean radial velocities and the largest number of valid gates along each radial. Because the near-zero radial velocity typically appears at two radials (the two local minima marked by L1 with 382 valid gates and L2 with 547 valid gates in Fig. 1c), the radial with more valid range gates (L2) is selected as the first radial, which is at $\sim 260^{\circ}$, as shown by the yellow line in Fig. 1a.

The above scheme in CIDA, originally described in HE2012a, works well in determining the proper first radial in most situations but tends to fail when multiplealiased velocities appear in some typhoon or hurricane cases. As an example, Fig. 1b shows a failure case from an S-band Taiwan radar system with an elevation angle of $19.5^{\circ}$ at 2208 UTC 18 September 2010 from the Typhoon Fanapi observations from the radar located in Hualian, Taiwan. More than $80 \%$ of the radial velocities are aliased at this elevation. The yellow line (corresponding to L1 in Fig. 1d) is not the best first reference radial found using the original method in CIDA, which was due to the second aliasing, which resulted in small values of aliased velocities between the $30^{\circ}$ and $45^{\circ}$ azimuth angles.

To eliminate the ambiguities caused by the contaminations of the radial velocity data, we added a criterion in ADTH based on the prevailing environmental wind direction. The wind is estimated using the GVAD method developed by Gao et al. (2004). The classic VAD technique (Browning and Wexler 1968) is able to estimate the areal mean vertical profile of the horizontal wind above a Doppler radar. However, the classic VAD technique encounters difficulty when the radial velocities are aliased or have large areas of missing data. The GVAD technique uses velocity gradient information to retrieve the wind speed and direction from radial velocity observations without the need for prior dealiaising and other measurements. The GVAD technique can calculate the wind components $u_{0}$ and $v_{0}$ at each level $z_{0}$ for a constant scan elevation angle through the minimization of the following cost function:

$$
\begin{aligned}
J\left(u_{0}, v_{0}\right)= & \sum_{n=1}^{N} \frac{1}{\sigma_{n}^{2}}\left\{\left(\frac{\partial V_{o}}{\partial \varphi}\right)_{n}-\cos \theta_{n}\right. \\
& \left.\times\left[\left(\cos \varphi_{n}\right) u_{0}-\left(\sin \varphi_{n}\right) v_{0}\right]\right\}^{2},
\end{aligned}
$$

where $\varphi$ is the azimuth angle, $\theta$ is the elevation angle, $\sigma_{n}=0.8$ represents the quality of the fit by calculating 

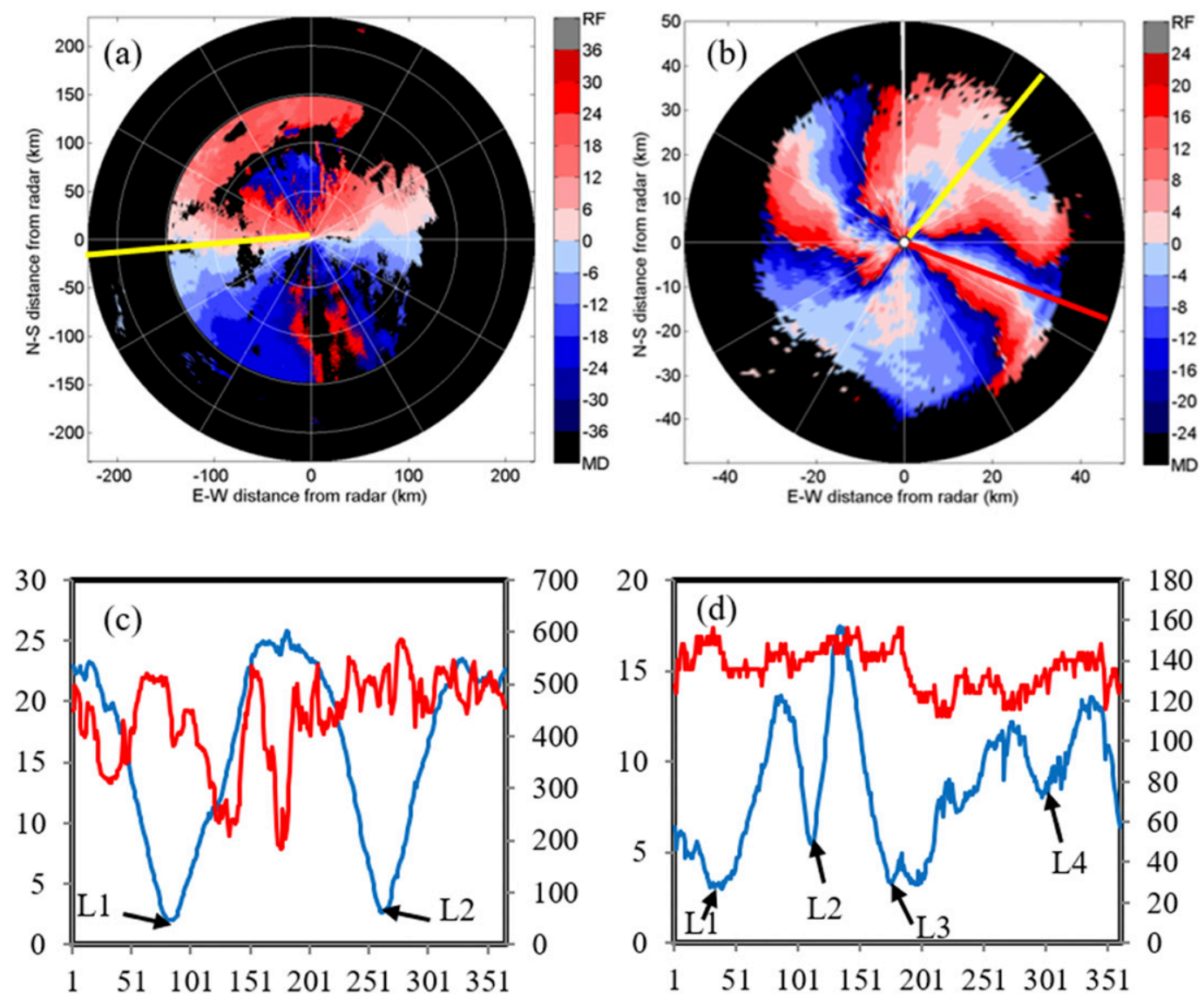

FIG. 1. Raw radar radial velocity (a) at $1.5^{\circ}$ elevation angle from the CINRAD S-band radar located in Wenzhou, China, at 1045 UTC $29 \mathrm{Jul} 2008$ and (b) at $19.5^{\circ}$ elevation angle from the S-band radar located at Hualian at 2208 UTC 18 Sep 2010. MD is the abbreviation of missing data observation. The yellow lines are the first reference radial using the method in HE2012a, and the red line is the reference radial calculated by the GVAD method. (c),(d) The radial-averaged mean radial velocity with respect to azimuth angle (blue curve with north as $0^{\circ}$, left $y$ axis, and $\mathrm{m} \mathrm{s}^{-1}$ ) and the total number of the valid gates used for the computation of the mean (red curve, right $y$ axis) for (a) and (b), respectively.

the dispersion of the data around the straight line, and $n$ is the index of the data in the azimuthal direction (Tabary et al. 2001). The calculation of the azimuthal gradients of radial velocity is similar to Eq. (11) in Gao et al. (2004) and is done by taking the difference of the velocity at adjacent points in the azimuthal direction,

$$
\left(\frac{\partial V_{o}}{\partial \varphi}\right)_{n}=\left(\frac{V_{o}^{n+1}-V_{o}^{n}}{\varphi^{n+1}-\varphi^{n}}\right)
$$

Now we illustrate how the GVAD wind is used as an additional criterion in determining the first radial using the example shown in Fig. 1b. In the first step, four radials with local minima, marked by L1, L2, L3, and L4, respectively, in Fig. 1d, were determined. Among the four local minima, L1 was chosen as a candidate first radial because it had the smallest average radial velocity and the largest number of valid data points. Using the least squares fit method in Eq. (3), the average wind direction over all the vertical levels for the elevation angle in Fig. $1 \mathrm{~b}$ is found to be $202.7^{\circ}$ (northward wind is $0^{\circ}$ here). Hence, the zero lines should be near $112.7^{\circ}$ or $292.7^{\circ}$ (perpendicular directions to that of the wind), which correspond to the two local minima, L2 and L4, in Fig. 1d. We choose L2 as the possible first radial because it has a smaller mean radial velocity than L4 (see Fig. 1d). For the final step to determine whether L1 or L2 is the real zero line, the following criterion is used. If the difference between L1 and L2 is less than $20^{\circ}$ (empirically determination), suggesting that L1 is near the zero line, then it is safe to choose the radial L1 as the first radial; otherwise, the radial L2 is selected.

The GVAD wind criterion is necessary for correctly dealiasing typhoon and hurricane cases. For more details on the basic procedure of the first radial selection, the reader is referred to HE2012a. 


\section{b. Module 2: Multipass dealiasing}

A TWO-WAY DEALIASING PROCEDURE IN BOTH RADIAL AND AZIMUTHAL DIRECTIONS TO IMPROVE THE DEALIASING RELIABILITY, ESPECIALLY WHEN TYPHOON/HURRICANE EYE IS VERY CLOSE TO RADAR STATION

Dealiasing starts with the radials next to both sides of the first reference radial chosen by module 1 in the two passes: one in the clockwise direction and the other in the counterclockwise direction. Each pass goes through $180^{\circ}$. In each of the half circles, the module dealiases in the radial and azimuthal directions in a two-way fashion (see the illustration in Fig. 2). The procedure in ADTH is similar to that in HE2012a but with the additional steps along the azimuthal direction (Figs. $2 \mathrm{c}$ and $2 \mathrm{~d}$ ). For each gate, the dealiasing is performed using a continuity check that compares its radial velocity with a reference radial velocity, which is selected from the dealiased velocities in the preceding gates. For the details of how to select the reference radial velocity, the reader is referred to HE2012a. This two-way and two-direction (radial and azimuthal) dealiasing procedure does not have the dependence of sounding or VAD wind profiles and is effective for dealing with near-range aliasing and aliasing next to missing gates.

\section{c. Module 3: Local error check and dealiasing}

\section{A LOCAL ERROR CHECK AND A LINEAR SQUARE ERROR CHECK IN RADIAL DIRECTION AND A QUADRATIC LEAST SQUARES ERROR CHECK IN AZIMUTHAL DIRECTION TO CLEAN UP THE REMAINING DEALIASING ERRORS}

A local error check is performed as a final step in ADTH to clean up any local errors that may still exist after the abovementioned two modules. At each gate, an average velocity of $\bar{V}$ over all the valid points $k$ within a specified geometrical window is calculated as the reference wind for the following equation to compute a suitable $n$ in Eq. (2):

$$
n=\operatorname{NINT}\left(\frac{\bar{V}-V_{o}}{2 V_{\max }}\right),
$$

where NINT represents rounding off to the nearest integer. The size of the geometrical window is defined by the number of gates $k$, which is empirically chosen and varies with the range distance from a radar station. The size of the window is set to $9 \times 9$ and $15 \times 15$ for distances between the radar station and the observation gate less than and greater than $100 \mathrm{~km}$, respectively. The local error checks and dealiasing are not performed when the number of valid points in the specified window is less than $70 \%$ (empirically determined) of the total gates to avoid uncertainty.

The above local dealiasing scheme works successfully for most weather situations, but it may fail when the eye of a hurricane or typhoon is close to the radar station. Therefore, the following two steps are added in ADTH to ensure the robustness of the algorithm. First, a linear least squares error check in the radial direction is carried out. The velocity is assumed to satisfy a linear relationship along each radial: $V_{T}=a_{m} \times X_{n}+b_{m}$, where $V_{T}$ is the true radial velocity and $X_{n}$ is the distance between a range gate and the radar station. The constants $a_{m}$ and $b_{m}$ are obtained by solving a linear least squares equation using the observed radial velocities on that radial. All the velocity values that do not fit this constraint well are then dealiased using Eq. (2). The second step is to perform a quadratic least squares error check in the azimuthal direction. Since the measured radial velocity is the wind velocity projected onto the radial direction, in each half circle, starting from the first radial $l_{1}$ and extending to the radial $l_{2}\left(180^{\circ}\right.$ away from $\left.l_{1}\right)$, all the velocities with the same range from the radar in the azimuthal direction approximately satisfy a quadratic relationship (the sine function between $0^{\circ}$ and $\pm \pi$ ). Thus, a quadratic least squares fit is used to identify and correct any remaining aliased data.

\section{Results of ADTH applications}

The dealiasing algorithm ADTH described above was tested on four typhoon cases and two hurricane cases. These six cases were observed by S-band radar from different radar stations. The radial velocity observations covered a radius of $230 \mathrm{~km}$ for the S-band radar systems. Depending on the PRFs of the different radars, the Nyquist velocity ranged from 21.15 to $31.61 \mathrm{~m} \mathrm{~s}^{-1}$. The ADTH's continuous dealiasing covered a period close to $12 \mathrm{~h}$ for each case. And more than half of the raw volumes contained aliased velocities in each selected case. The total number of volumes was 789 and the number of constant-elevation scans was 4847. Table 1 presents the total volumes, the total constantelevation scans, and the total valid gates of the six selected periods. Table 1 also shows the number of volumes containing data with aliased velocities and the number of gates with aliased velocities in raw data in each period, of which $75 \%$ of volumes have aliased velocities and $44.9 \%$ of the gates contain aliased velocities between the six selected cases. The dealiasing results for these cases are shown below and are followed by a statistical evaluation.

\section{a. Result of typhoon case}

Typhoon Fanapi formed east of the Philippines, moved westward, and struck Taiwan on 19 September 2010. 
(a)

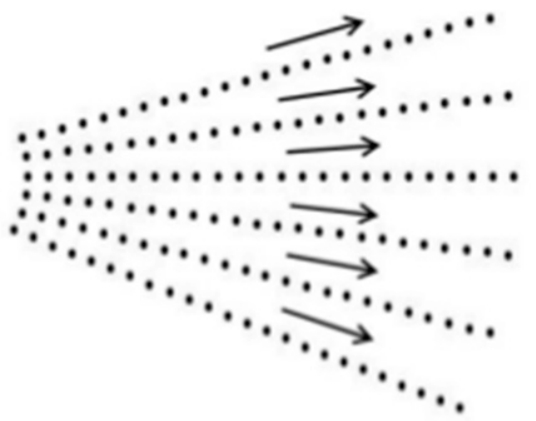

(c)

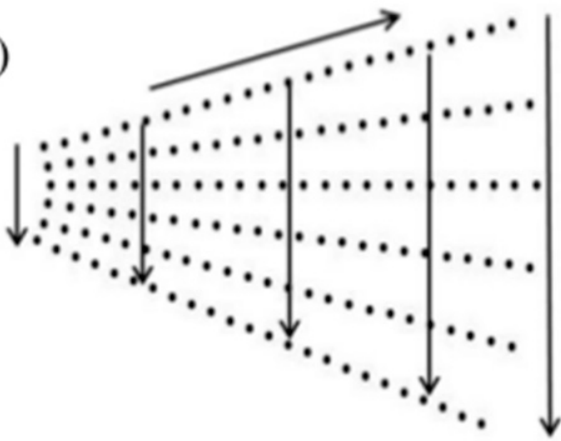

(b)

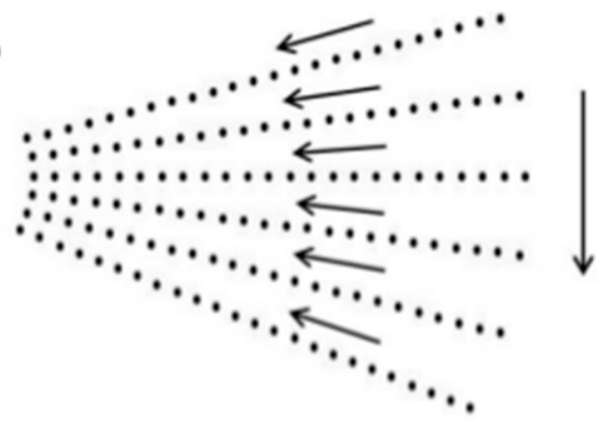

(d)

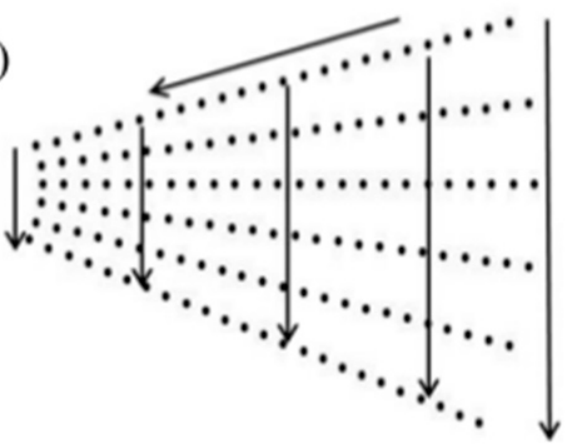

FIG. 2. An illustration of dealiasing along the radial direction (a) from inner to outer range gate and (b) from outer to inner range gate, and along the azimuthal direction proceeding (c) from inner to outer range gate and (d) from outer to inner range gate.

It was notable because of the severe property damage and five lost lives that occurred in Taiwan alone as a result of heavy rainfall, landslides, and floods. With a maximum sustained wind speed of $54 \mathrm{~m} \mathrm{~s}^{-1}$, some Taiwan radar systems experienced double aliasing, which made the dealiasing more challenging. Another challenge for typhoon dealiasing can occur when a typhoon eye is close to a radar station because the radial velocity field exhibits asymmetry at the two sides of the zero-velocity line.

The S-band radar located in Hualian near the eastern coast of Taiwan has a relatively low Nyquist velocity of $21.15 \mathrm{~m} \mathrm{~s}^{-1}$ and was close to the typhoon eye at 2208 UTC 18 September 2010, right before Fanapi made landfall. Therefore, it provides good datasets to test ADTH's capability in extreme circumstances. Figures $3 a, 3 c$, and $3 e$ show the raw radial velocities observed by the Hualien radar at the elevation angles of $1.5^{\circ}, 4.3^{\circ}$, and $19.5^{\circ}$, respectively. Because of the blocking by the mountains near the coastline of Taiwan, the Hualien radar operates by only partial scanning over the elevation angles between $0.5^{\circ}$ and $9.9^{\circ}$ and fully scanning the two high-elevation angles above. The partial scanning coverage of the wind fields at low-elevation angles can cause particular difficulties for any dealiasing algorithms. As seen in Fig. 3, the eye of Fanapi was only $50 \mathrm{~km}$ east of the radar at this time. The velocity observations to the northwest and southwest of the eye of the typhoon show considerable twice-aliased data. ADTH correctly detected the first radial near the zero line and corrected the twice-aliased data. Figure $3 b$ is the final dealiased result at a $1.5^{\circ} \mathrm{el}-$ evation angle after all of the dealiasing modules were run (the color scales are different between before and after dealiasing of this case). At the $4.3^{\circ}$ elevation angle, more than $90 \%$ of the valid radial velocities were aliased and there exists a discontinuous echo to the north of the typhoon eye as shown in Fig. 3c. For this elevation angle, the local dealiasing method based on the least squares error check described in the last module played an important role. As shown in Fig. 3d, ADTH successfully corrected the severely aliased velocity data. For the elevation angle of $19.5^{\circ}$, the GVAD technique in module 2 proved useful in correctly finding the first reference radial near $292^{\circ}$ in Fig. 3e, as detailed in the description of Fig. 1 in section 2 . The correct first reference radial ensured the rest of the modules were executed successfully, resulting in the successful dealiasing of this angle as shown in Fig. 3f.

\section{b. Result of hurricane case}

Hurricane Isaac made landfall in southeastern Louisiana on 21 August 2012. It caused extensive heavy rainfall and inland flooding over southern Mississippi and southeastern Louisiana. Isaac was estimated to be directly responsible for five deaths in the United States. 
The S-band KLIX radar, located in New Orleans, Louisiana, at 2043 UTC 28 August 2012, right before Isaac made landfall near Louisiana, provided good datasets to evaluate ADTH's performance when the gates with aliased velocities are surrounded by gates with missing data, which causes difficulty for any dealiasing algorithm. Figure $4 \mathrm{a}$ shows the raw radial velocity at the elevation angle of $0.5^{\circ}$. In spite of the existence of the hurricane eye and the discontinuous velocity data near the aliased area to the south and southeast of the radar, the new algorithm performs very well by correctly dealiasing two areas in Fig. $4 \mathrm{~b}$. Figures $4 \mathrm{c}$ and $4 \mathrm{~d}$ show the raw radial velocity and dealiased radial velocity, respectively, at the $4.3^{\circ}$ elevation angle at the same observation time. Again, the dealiased velocity field is clean and without noticeable errors.

The above results from some selected scans demonstrated that the new ADTH algorithm has the capability to address challenging aliasing issues in hurricane situations. The overall statistics for both hurricane cases over a period of $12 \mathrm{~h}$ are computed, and the results will be discussed in the next subsection.

\section{c. Comparison with the original CIDA via statistical evaluations}

The skill of ADTH over all the tested radar volumes from the four typhoon cases and the two hurricane cases was evaluated statistically and compared with that of CIDA, as described in HE2012a. The evaluation was based on the percentage of gates with correct velocity data after running two different algorithms. The period of continuous dealiased data is close to $12 \mathrm{~h}$ for each case. There are $75 \%$ raw observed volumes containing gates with aliased velocities in all the selected periods, which means most of the volumes contain velocity data that need to be dealiased. All of the constant-elevation scans were examined, and the fields with aliased velocities were manually edited to produce "truths" for evaluation. The truths radial velocities were manually edited by the National Center for Atmospheric Research (NCAR) SOLO II package (HE2012a). Since the performance statistics depend on the accuracy of the manual dealiasing process, we carefully checked the manual editing results to detect and correct any possible mistakes. Given our expertise and experience in the area, we are confident that the manual editing results are reliable.

Table 2 shows the statistical dealiasing results indicating the percentage of all correct gates after dealiasing out of all gates with valid data for the six cases using ADTH and CIDA. It is shown that ADTH outperforms CIDA for each case. The Taiwan Hualian radar for the Typhoon Fanapi case and the China Haikou radar for the Typhoon Rammasun case had the largest skill increase, which was mainly attributable to the improved detection of the first radial and special local error 

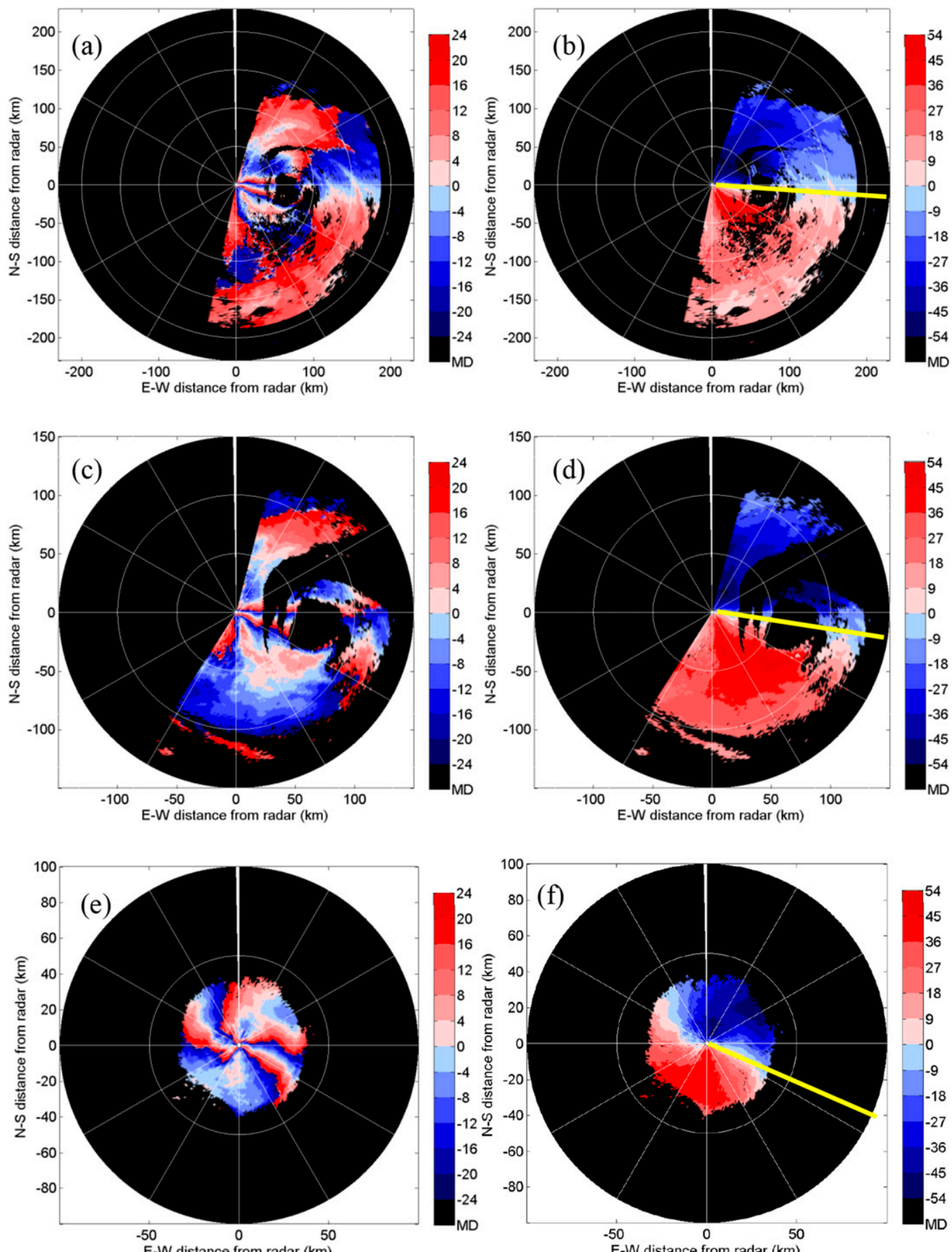

FIG. 3. Radar radial velocity from the Hualien radar of the Taiwan radar network at 2208 UTC 18 Sep 2010. (a) Raw velocity at $1.5^{\circ}$ elevation angle, (b) dealiased result at $1.5^{\circ}$ elevation angle, (c) raw velocity at $4.3^{\circ}$ elevation angle, (d) dealiased result at $4.3^{\circ}$ elevation angle, (e) raw velocity at $19.5^{\circ}$ elevation angle, and (f) dealiased result at $19.5^{\circ}$ elevation angle. The Nyquist velocity for the Hualien radar is $21.15 \mathrm{~m} \mathrm{~s}^{-1}$. The $x$ axis is the distance away from the radar in the west-east direction, while the $y$ axis is the distance away from the radar in the south-north direction. The spatial scales of the pairs of images at different elevations are different. The yellow lines are the first reference radials. 

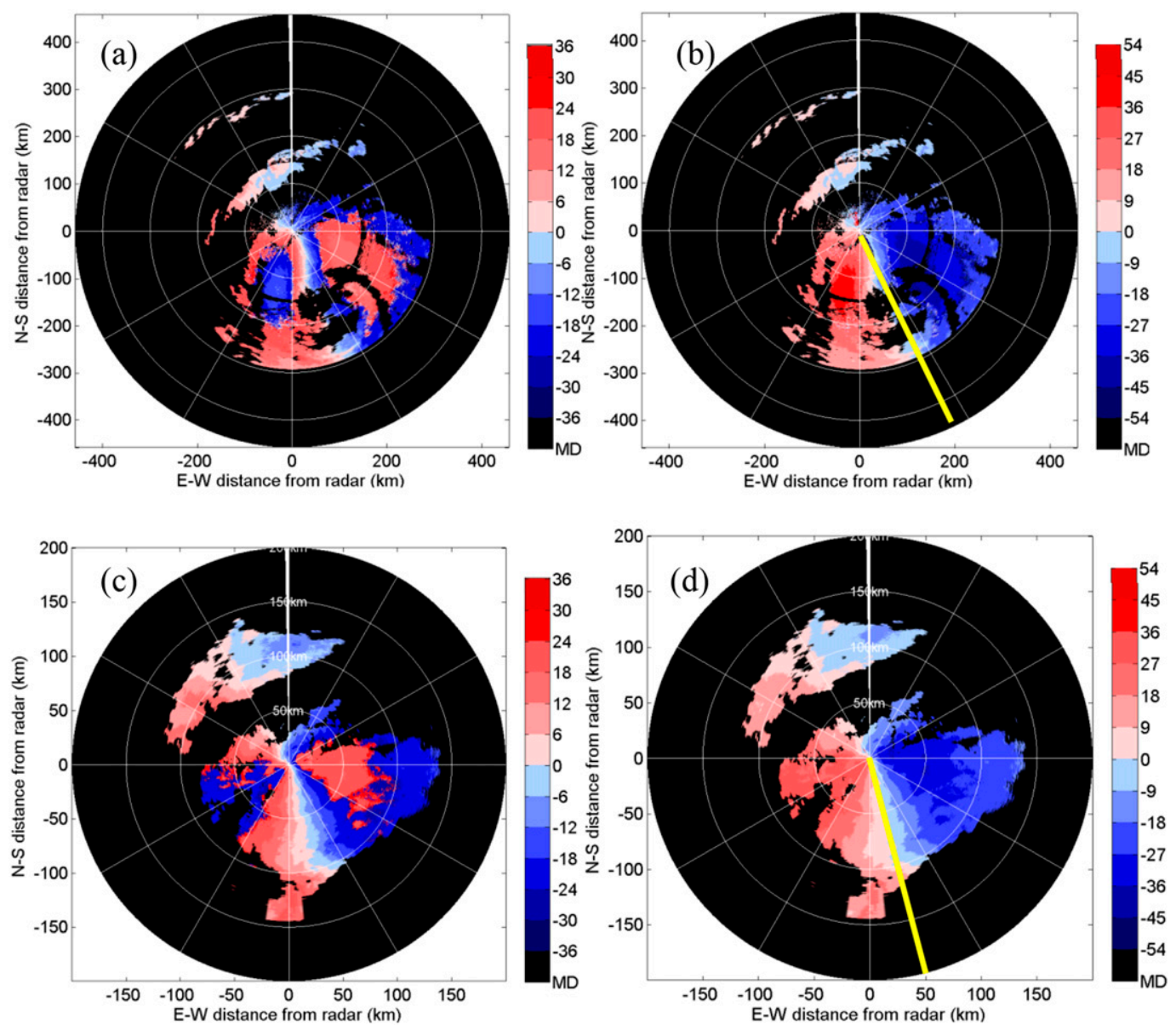

FIG. 4. Radar radial velocity from KLIX of NEXRAD at 2043 UTC 28 Aug 2012. (a) Raw velocity at $0.5^{\circ}$ elevation angle, (b) dealiased result at $0.5^{\circ}$ elevation angle, (c) raw velocity at $4.3^{\circ}$ elevation angle, and (d) dealiased result at $4.3^{\circ}$ elevation angle. The spatial scales of the pairs of images at different elevations are different. The yellow lines are the first reference radials.

checks. And more than half of the aliased data are twice aliased, which the CIDA cannot deal with well in these two cases. Comparing the statistical results of the six cases, the new ADTH algorithm can dealias more than $98 \%$ of the radial velocity correctly. It has a reliable performance for the typhoon and hurricane systems analyzed here.

\section{Summary and conclusions}

In this paper we presented and evaluated the velocity dealiasing algorithm $\mathrm{ADTH}$, which was developed as an improvement to CIDA (operational on the Chinese radar network) with the aim of dealiasing typhoon and hurricane systems. The performance of the ADTH algorithm was examined for four typhoon cases and two hurricane cases observed by S-band radars stations, totaling 789 volumes or 7981 constant-elevation velocity fields. The selected cases had raw velocities that were severely aliased for a large percentage of the constantelevation scans, and some included challenging dealiasing situations with multiple-aliased radial velocities as a result of strong wind speeds.

TABLE 2. List of all six cases and the associated dealiasing results (the percentage of all correct gates after dealiasing out of all valid gates) using the ADTH algorithm and the original CIDA algorithm; H: hurricane, T: typhoon.

\begin{tabular}{lcccccr}
\hline \hline Radar site & KLIX $(\mathrm{H})$ & KAKQ $(\mathrm{H})$ & Hualien $(\mathrm{T})$ & Haikou $(\mathrm{T})$ & Wenzhou $(\mathrm{T})$ & Xiamen $(\mathrm{T})$ \\
\hline ADTH & $99.04 \%$ & $99.13 \%$ & $98.32 \%$ & $98.91 \%$ & $99.62 \%$ & $99.54 \%$ \\
CIDA & $97.36 \%$ & $96.68 \%$ & $88.79 \%$ & $80.33 \%$ & $93.11 \%$ & $99.02 \%$ \\
\hline
\end{tabular}


The new ADTH comprises three modules that were designed to determine the starting reference radial, to identify and modify the aliased velocity gates with a twoway multipass scheme and to rectify the incorrect results with a final local error check. The selection of the first radial near the zero-velocity line ensures that the dealiasing algorithm starts from velocity data that are less likely aliased. The GVAD technique, using the velocity gradient information in module 1 , was shown to help improve the accuracies of the first radial detections in the typhoon and hurricane cases where the multiplealiased situations occur rather frequently. The two-way multipass dealiasing method implemented in module 2 improved the accuracy of the dealiasing, especially when the aliasing occurs near large areas of missing data. In addition, we showed that the additional linear least squares error check in the radial direction and quadratic least squares error checks in the azimuthal direction were able to eliminate the remaining dealiasing errors in the typhoon and hurricane systems.

The statistical evaluation of ADTH, using all correct gates after dealiasing out of all valid gates, demonstrated that the upgraded ADTH outperformed the preceding algorithm of CIDA for all six cases, with largest improvements for the Taiwan Typhoon Fanapi case and for the China Typhoon Rammasun case, which contain lots of twice-aliased observed velocity gates. The improvement resulted from more accurate detections of the first radial and local error checks. The success rate of ADTH for the typhoon and hurricane systems was above $98 \%$.

ADTH is developed for dealiasing typhoon and hurricane systems and is applied only when these types of storms are already present. The fact is that practical and operational application of the ADTH requires human intervention or a special algorithm that will help to determine the typhoon and hurricane storms. Lee and Marks (2000) and Lee et al. (2014) have developed a GBVTDsimplex algorithm that can reduce the uncertainties in estimating the TC center position and improves the quality of the GBVTD-retrieved TC circulation. The GBVTDsimplex algorithm is computationally efficient for real-time applications. Such algorithms like the GBVTD-simplex algorithm can be used to identify typhoon and hurricane storms and to decide whether to execute ADTH for dealiasing. ADTH is being implemented for the Variational Doppler Data Analysis System (VDRAS) and the Weather Research and Forecasting Data Assimilation (WRFDA) system as one of the radar quality control software systems. Both systems have operational capabilities, so ADTH can be tested operationally in the future.

Acknowledgments. This work was supported by the Research Program for Nanjing Joint Center of Atmospheric
Research (NJCAR2016MS01), the China Special Fund for Meteorological Research in the Public Interest (Grant GYHY 201506004), the Research Program for Key Laboratory of Meteorology and Ecological Environment of Hebei Province (Z201603Z), Key Laboratory of South China Sea Meteorological Disaster Prevention and Mitigation of Hainan Province (Grant SCSF201804), and the Priority Academic Program Development of Jiangsu Higher Education Institutions (PAPD).

\section{REFERENCES}

Bergen, W. R., and S. C. Albers, 1988: Two- and three-dimensional dealiasing of Doppler radar velocities. J. Atmos. Oceanic Technol., 5, 305-319, https://doi.org/10.1175/1520-0426(1988) 005<0305:TATDDA $>2.0$. CO 2 .

Browning, K. A., and R. Wexler, 1968: The determination of kinematic properties of a wind field using Doppler radar. J. Appl. Meteor., 7, 105-113, https://doi.org/10.1175/1520-0450(1968) 007<0105:TDOKPO > 2.0.CO;2.

Eilts, M. D., and S. D. Smith, 1990: Efficient dealiasing of Doppler velocities using local environment constraints. J. Atmos. Oceanic Technol., 7, 118-128, https://doi.org/10.1175/1520-0426(1990) 007<0118:EDODVU>2.0.CO;2.

Gao, J., K. K. Droegemeier, J. Gong, and Q. Xu, 2004: A method for retrieving mean horizontal wind profiles from single-Doppler radar observations contaminated by aliasing. Mon. Wea. Rev., 132, 1399-1409, https://doi.org/10.1175/1520-0493-132.1.1399.

Gong, J., L. Wang, and Q. Xu, 2003: A three-step dealiasing method for Doppler velocity data quality control. J. Atmos. Oceanic Technol., 20, 1738-1748, https://doi.org/10.1175/1520-0426(2003) $020<1738$ :ATDMFD>2.0.CO;2.

He, G., G. Li, X. Zou, and P. S. Ray, 2012a: Applications of a velocity dealiasing scheme to data from China New Generation Weather Radar System (CINRAD). Wea. Forecasting, 27, 218-230, https://doi.org/10.1175/WAF-D-11-00054.1.

,,$-- \ldots$, and $-2012 \mathrm{~b}$ : A velocity dealiasing scheme for synthetic C-band data from China's New Generation Weather Radar System (CINRAD). J. Atmos. Oceanic Technol., 29, 1263-1274, https://doi.org/10.1175/JTECH-D-12-00010.1.

Hu, M., M. Xue, and K. Brewster, 2006: 3DVAR and cloud analysis with WSR-88D level-II data for the prediction of the Fort Worth, Texas, tornadic thunderstorms. Part I: Cloud analysis and its impact. Mon. Wea. Rev., 134, 675-698, https://doi.org/10.1175/MWR3092.1.

James, C. N., and R. A. Houze Jr., 2001: A real-time fourdimensional Doppler dealiasing scheme. J. Atmos. Oceanic Technol., 18, 1674-1683, https://doi.org/10.1175/1520-0426 (2001)018<1674:ARTFDD>2.0.CO;2.

Jiang, Y., and Q. Xu, 2016: Adaptive dealiasing for Doppler velocities scanned from hurricanes and typhoons. J. Atmos. Oceanic Technol., 33, 1931-1947, https://doi.org/10.1175/JTECH-D-15-0146.1.

Jing, Z., and G. Wiener, 1993: Two-dimensional dealiasing of Doppler velocities. J. Atmos. Oceanic Technol., 10, 798-808, https://doi.org/ 10.1175/1520-0426(1993)010<0798:TDDODV > 2.0.CO;2.

Lee, W., and F. D. Marks, 2000: Tropical cyclone kinematic structure retrieved from single-Doppler radar observations. Part II: The GBVTD-simplex center finding algorithm. Mon. Wea. Rev., 128, 1925-1936, https://doi.org/10.1175/1520-0493 (2000)128<1925:TCKSRF $>2.0$. CO;2.

, X. Tang, and B. J. Jou, 2014: Distance velocity-azimuth display (DVAD) - New interpretation and analysis of Doppler 
velocity. Mon. Wea. Rev., 142, 573-589, https://doi.org/10.1175/ MWR-D-13-00196.1.

Lim, E., and J. Sun, 2010: A velocity dealiasing technique using rapidly updated analysis from a four-dimensional variational Doppler radar data assimilation system. J. Atmos. Oceanic Technol., 27, 1140-1152, https://doi.org/10.1175/2010JTECHA1300.1.

Merritt, M. W., 1984: Automatic velocity dealiasing for real-time applications. Proc. 22nd Conf. on Radar Meteorology, Zurich, Switzerland, Amer. Meteor. Soc., 528-533.

Ray, P. S., and C. Ziegler, 1977: De-aliasing first-moment Doppler estimates. J. Appl. Meteor., 16, 563-564, https://doi.org/ 10.1175/1520-0450(1977)016<0563:DAFMDE >2.0.CO;2.

Simonin, D., S. P. Ballard, and Z. Li, 2014: Doppler radar radial wind assimilation using an hourly cycling 3D-Var with a $1.5 \mathrm{~km}$ resolution version of the Met Office Unified Model for nowcasting. Quart. J. Roy. Meteor. Soc., 140, 2298-2314, https://doi.org/10.1002/qj.2298.

Sun, J., 2005: Initialization and numerical forecasting of a supercell storm observed during STEPS. Mon. Wea. Rev., 133, 793-813, https://doi.org/10.1175/MWR2887.1.

- , and Y. Zhang, 2008: Analysis and prediction of a squall line observed during IHOP using multiple WSR-88D observations. Mon. Wea. Rev., 136, 2364-2388, https://doi.org/10.1175/ 2007MWR2205.1.

Tabary, P., G. Scialom, and U. Germann, 2001: Real-time retrieval of the wind from aliased velocities measured by Doppler radars.
J. Atmos. Oceanic Technol., 18, 875-882, https://doi.org/10.1175/ 1520-0426(2001)018<0875:RTROTW >2.0.CO;2.

Wang, M., K. Zhao, W.-C. Lee, B. Jong-Dao Jou, and M. Xue, 2012: The gradient velocity track display (GrVTD) technique for retrieving tropical cyclone primary circulation from aliased velocities measured by single-Doppler radar. J. Atmos. Oceanic Technol., 29, 1026-1041, https://doi.org/ 10.1175/JTECH-D-11-00219.1.

Xiao, Q., and J. Sun, 2007: Multiple-radar data assimilation and short-range quantitative precipitation forecasting of a squall line observed during IHOP_2002. Mon. Wea. Rev., 135, 3381-3404, https://doi.org/10.1175/MWR3471.1.

Xu, Q., K. Nai, and L. Wei, 2010: Fitting VAD winds to aliased radial-velocity observations: A global minimization problem in the presence of multiple local minima. Quart. J. Roy. Meteor. Soc., 136, 451-261, https://doi.org/10.1002/qj.589.

, P. Zhang, S. Liu, and D. Parrish, 2011: A VADbased dealiasing method for radar velocity data quality control. J. Atmos. Oceanic Technol., 28, 50-62, https://doi.org/ 10.1175/2010JTECHA1444.1.

_- Y. Jiang, and L. Liu, 2014: Fitting parametric vortices to aliased Doppler velocities scanned from hurricanes. Mon. Wea. Rev., 142, 94-106, https://doi.org/10.1175/MWR-D-12-00362.1.

Zhang, J., and S. Wang, 2006: An automated 2D multipass Doppler radar velocity dealiasing scheme. J. Atmos. Oceanic Technol., 23, 1239-1248, https://doi.org/10.1175/JTECH1910.1. 